\title{
Surface energetics, pit formation, and chemical ordering in InGaN alloys
}

Cite as: Appl. Phys. Lett. 74, 2319 (1999); https://doi.org/10.1063/1.123837

Submitted: 11 December 1998 . Accepted: 15 February 1999. Published Online: 14 April 1999

J. E. Northrup, L. T. Romano, and J. Neugebauer

\section{ARTICLES YOU MAY BE INTERESTED IN}

Structural origin of V-defects and correlation with localized excitonic centers in InGaN/GaN multiple quantum wells

Applied Physics Letters 72, 692 (1998); https://doi.org/10.1063/1.120844

Formation of V-shaped pits in InGaN/GaN multiquantum wells and bulk InGaN films Applied Physics Letters 73, 1634 (1998); https://doi.org/10.1063/1.122229

Solid phase immiscibility in GalnN

Applied Physics Letters 69, 2701 (1996); https://doi.org/10.1063/1.117683

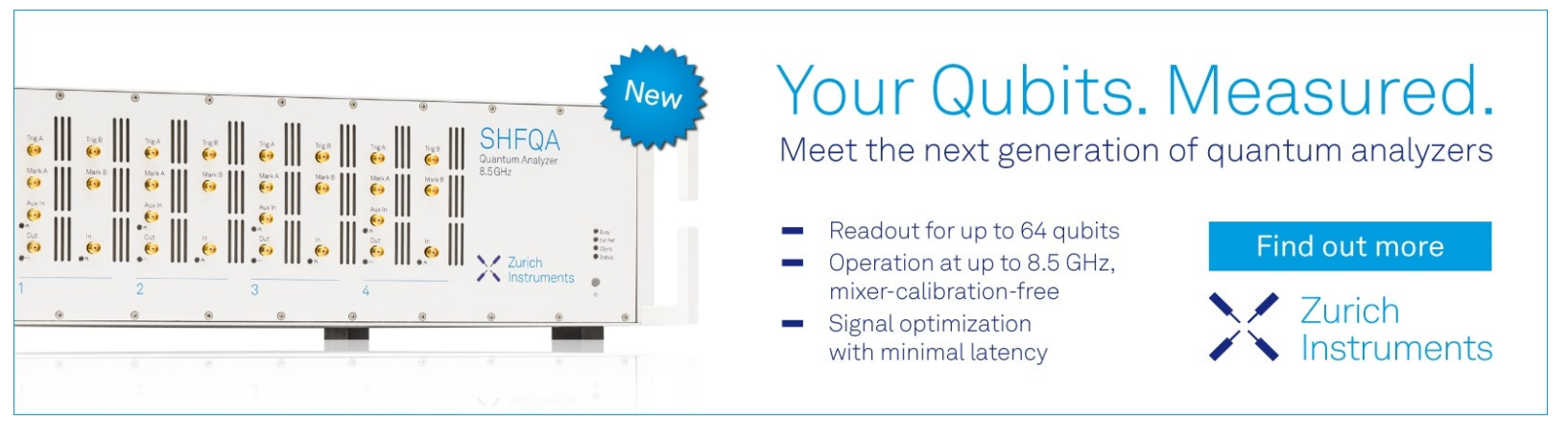




\title{
Surface energetics, pit formation, and chemical ordering in InGaN alloys
}

\author{
J. E. Northrup a) and L. T. Romano \\ Xerox Palo Alto Research Center, 3333 Coyote Hill Road, Palo Alto California, 94304 \\ J. Neugebauer \\ Fritz-Haber-Institut der Max-Planck-Gesellschaft, Faradayweg 4-6, D-14195 Berlin Germany
}

(Received 11 December 1998; accepted for publication 15 February 1999)

\begin{abstract}
We present first-principle calculations of the structure and energetics of the $\mathrm{GaN}(1011)$ surface, and present models for the reconstructions. A strong preference for In surface segregation and occupation of specific surface sites is demonstrated. We argue that inverted pyramid defect formation is enhanced by segregation of In on (1011) facets. We propose that the chemical ordering recently observed in InGaN alloys is driven by the preference for In incorporation at the sites of reduced $\mathrm{N}$ coordination present at step edges during growth on the (0001) and (0001) surfaces.

(C) 1999 American Institute of Physics. [S0003-6951(99)02015-X]
\end{abstract}

The desire to understand and control epitaxial growth of GaN films has motivated a number of studies of the structure and properties of GaN surfaces. ${ }^{1}$ Recently, the promise of lateral epitaxial overgrowth ${ }^{2,3}$ as a method for threading defect reduction has sparked interest in the GaN(1011) surface that is present as a sidewall facet in this growth mode. Additional motivation for studies of the (1011) surface stems from the presence of these facets in the inverted pyramid defects $^{4,5}$ that form at the termination of some threading dislocations on the GaN surface during growth of In-containing alloys. We have therefore conducted first-principle calculations of the atomic structure and energetics of clean and Incovered $\mathrm{GaN}(10 \underline{1} 1)$ surfaces.

The formation of InGaN quantum wells for blue laser devices requires the incorporation of In during epitaxial growth. A common extended defect occurring in these InGaN films is an inverted pyramid defect, or pit. ${ }^{4,5}$ Experimental analysis of the film morphology reveals that hexagonal shaped pits form where threading dislocations intersect the surface. The lateral extent of the pit at the surface increases with the thickness of the InGaN film and may be more than $100 \mathrm{~nm}$ at the surface of sufficiently thick InGaN films. The sides of the pits are (1011) surfaces. As will be discussed below, our calculations indicate that in the absence of In the equilibrium pit size is on the order of a few nanometers. However, we shall see that segregation of In on the (1011) surfaces can provide a strong driving force to reduce the surface energy and thereby allow formation of large inverted pyramid defects.

Recently, [0001] chemical ordering on the group III sublattice of InGaN alloys has been reported. ${ }^{6,7}$ We will show that this remarkable effect is a consequence of a preference for In incorporation on the sites of reduced $\mathrm{N}$ coordination that are present at step edges or on vicinal microfacets during growth.

The calculations are based on local density functional theory and employ first principles pseudopotentials and a plane wave basis as in previous work. ${ }^{1,8,9}$ The Ga $3 d$ and In $4 d$ electrons are included in the valence band. Full atomic

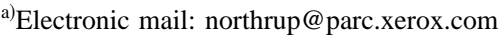

relaxation is included for all structures. The surfaces are modeled by repeated cells that typically contain $10 \mathrm{Ga}$ and $10 \mathrm{~N}$ atoms in each $1 \times 1$ unit cell. The plane wave cutoff is taken to be $60 \mathrm{Ry}$.

Schematic representations of some of the many structures considered are shown in Fig. 1. The structure that is stable under Ga-rich conditions is shown in Figs. 1(a) and 1(b). It is comprised of Ga atoms in two distinct types of sites in the surface layer. We refer to these sites as $B 2$ and $T 1$ sites. The atoms in $B 2$ sites are bonded to two $\mathrm{N}$ atoms in the layer below. This is similar to the bonding configuration of $\mathrm{Ga}$ atoms on the (001) ideal surface of cubic GaN. ${ }^{9}$ The atoms in the $T 1$ sites are bonded to one $\mathrm{N}$ atom in the layer below, as in the $1 \times 1 \mathrm{Ga}$ adlayer structure existing on the (0001) surface. ${ }^{1}$ As on the (001) and (0001) surfaces there is a strong interaction between the Ga atoms of the (1011) surface, and this helps to stabilize this reconstruction in $\mathrm{Ga}$ rich conditions, as shown in Fig. 2. The structure shown in Figs. 1(c) and 1(d) contains one Ga adatom per cell bonded in an $H 3$ site. This adatom is bonded to three $\mathrm{N}$ atoms in the

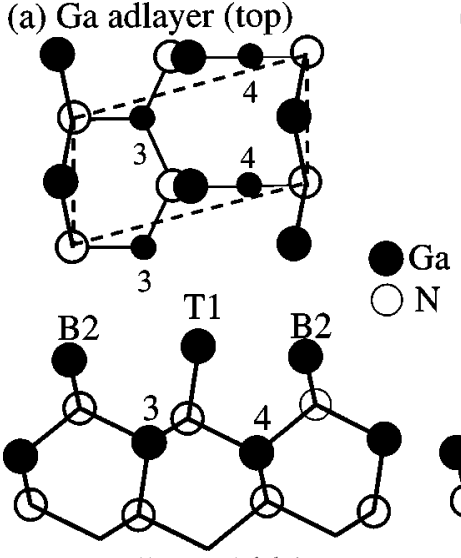

(b) Ga adlayer (side)
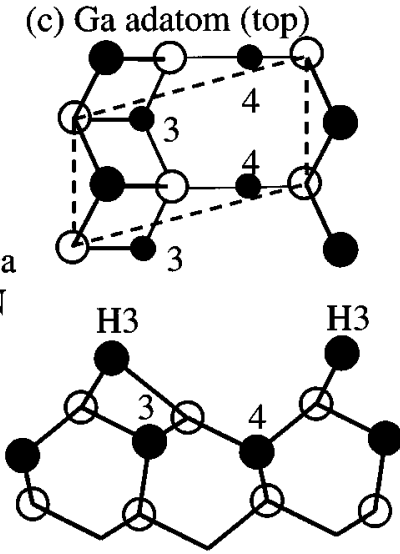

(d) $\mathrm{Ga}$ adatom (side)
FIG. 1. (a), (b) Representations of the GaN(1011) Ga adlayer surface. In sites $T 1$ and $B 2$ the Ga forms one or two bonds with $\mathrm{N}$ atoms. Sites 3 and 4 are near surface bulk sites. The dashed lines indicate the $1 \times 1$ cell boundary. (c), (d) Representation of the $1 \times 1 \mathrm{Ga}$ adatom structure that is stable in N-rich conditions. 


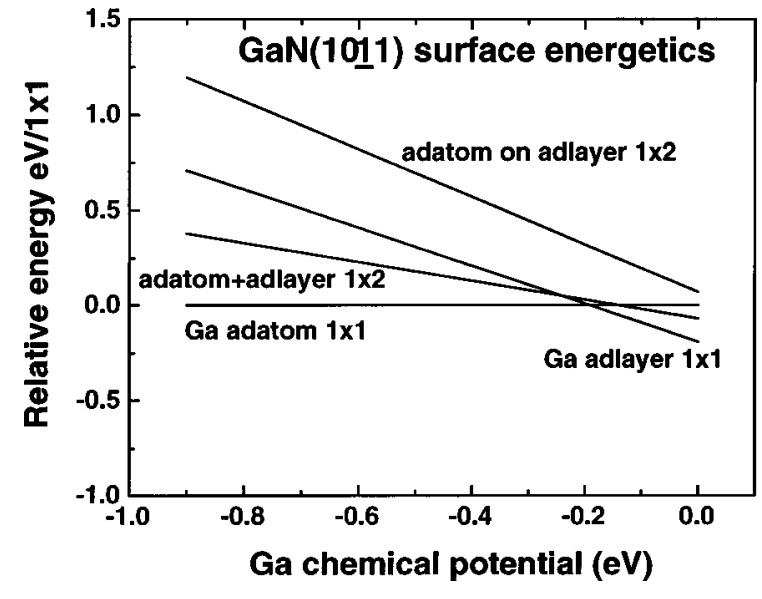

FIG. 2. Energetics of GaN(1011) surfaces as a function of the Ga chemical potential. The maximum equilibrium chemical potential of $\mathrm{Ga}$ is limited by precipitation of bulk Ga, i.e., $\mu_{\mathrm{Ga}}<\mu_{\mathrm{Ga}(\mathrm{bulk})} \equiv 0.0$.

layers below. As seen in Fig. 2, the adatom structure is found to be stable within a large region of the allowed range of $\mathrm{Ga}$ chemical potential. A structure with $1 \times 2$ periodicity is formed by adding $1 / 4$ monolayer of $\mathrm{Ga}$ atoms to the $1 \times 1$-adlayer structure, but this adatom-on-adlayer structure is not energetically favorable. A $1 \times 2$ mixture of the $1 \times 1$ adlayer and the $1 \times 1$ adatom structure is only slightly higher in energy than the average of the $1 \times 1$ adlayer and the 1 $\times 1$ adatom structures. Given the stability of the $1 \times 1 \mathrm{Ga}$ adlayer and the $1 \times 1 \mathrm{Ga}$ adatom models with respect to the other structures considered here, as well as their similarity to the structures that are stable on the (0001) and (001) surfaces, we think they are excellent models for the $\mathrm{GaN}(1011)$ surface.

We have also performed calculations in which one of the $\mathrm{Ga}$ atoms in a $1 \times 2$ supercell is replaced by an In atom. The replacement may occur on either a surface site $(B 2$ or $T 1)$ or in the bulk (site 3 or site 4 in Fig. 1). We find that it is energetically favorable to exchange In atoms in the bulk with $\mathrm{Ga}$ atoms at the surface: The energy reduction obtained in this exchange is $2.0 \mathrm{eV}$ for the $T 1$ site and $1.5 \mathrm{eV}$ for the $B 2$ site. (The results are independent of the bulk site, 3 or 4 .) This large energy reduction arising from In surface segregation is comparable to the energy required to create $\mathrm{GaN}$ surfaces. Consequently, In segregation may have a dramatic effect on the growth kinetics and morphology of InGaN films.

Let us now consider the question of the origin and equilibrium size of the hexagonal inverted pyramid defects indicated schematically in Fig. 3. Following Frank, ${ }^{10}$ we estimate the pit formation energy by using linear elasticity theory. The reference system is a crystal with a screw dislocation that threads to the surface. For specificity we calculate the energy with respect to that of a hollow-core screw dislocation with Burgers vector $\mathbf{b}=[0001]$. The Frank radius $r$ of such a dislocation is equal to $\mu b^{2} / 8 \pi^{2} \gamma_{0}$, where $\mu$ is the shear modulus and $\gamma_{0}$ is the surface energy of the internal surface. For $\gamma_{0}=110 \mathrm{meV} / \AA^{2}$, corresponding to the energy of the Ga-terminated (1010) surface, and $b=5.2 \AA$, corresponding to an elementary screw dislocation, $r=1.9 \AA .^{8,11}$ Removal of all atoms inside a cylinder of this radius leads to a hollow core defect with a bond topology identical to that obtained in atomistic calculations. ${ }^{12}$ Now the pit formation

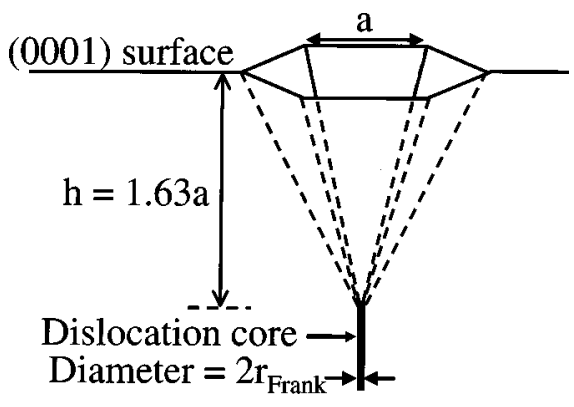

FIG. 3. A representation of a dislocation terminating in a pit at the (0001) surface. The depth of the pit is $h=1.63 a$. Pit formation creates six equivalent (1011) facets.

energy consists of three terms: (1) the change in surface energy resulting from replacing a hexagonal region of the (0001) surface with the six (1011) facets of the inverted pyramid, (2) the reduction in strain energy, and (3) the elimination of the dislocation core energy. The change in surface energy is equal to $S \gamma_{0} r^{2} x^{2}$, where $x=a / r$ is the size of the pit (measured in units of the Frank radius of the dislocation) and $S$ depends on the formation energies of the (0001) facet that is removed and (1011) facets that are formed. One may show that

$$
S=(3 \sqrt{ } 3 / 2)\left\{(1 / \alpha) \gamma(10 \underline{1} 1) / \gamma_{0}-\gamma(0001) / \gamma_{0}\right\},
$$

where $\alpha=0.468$ is the scalar product of the unit vectors that are normal to the (1011) and (0001) surfaces. ${ }^{13}$ The term corresponding to the reduction in strain energy is obtained by integrating the strain energy density $E(\rho)=\mu b^{2} / 8 \pi^{2} \rho^{2}$ over a cone of radius $a$ and height $(8 / 3)^{1 / 2} a$. The lower limit of the radial integration over $\rho$, the distance to the core, is $r$. The total formation energy of a pit of size $x$ is then given by

$$
\begin{aligned}
\Delta E(x) / \gamma_{0} r^{2}= & S x^{2}-2 \pi(8 / 3)^{1 / 2} \\
& \times[x /(x-1)](x \ln x-x+1)-\lambda x .
\end{aligned}
$$

The term $\lambda x$ represents the core energy of the dislocation. In our model $\lambda=2 \pi(8 / 3)^{1 / 2}$. The equilibrium pit size $x_{\min }$ is determined by minimization of $\Delta E(x)$, and the resulting values of $x_{\min }$ are plotted as function of $S$ in Fig. 4. Assuming that there is no strong dependence of the surface energies on orientation, one obtains $S \sim 3$. This assumption is justified by the results presented in Table I, where it is seen that the surface energies of GaN tend to cluster in the range between 110 and $125 \mathrm{meV} / \AA^{2}$. For $S \sim 3$ we obtain $x_{\min } \sim 5$, corresponding to a pit size of about $a \sim 10 \AA$. For $S \sim 2$ and with

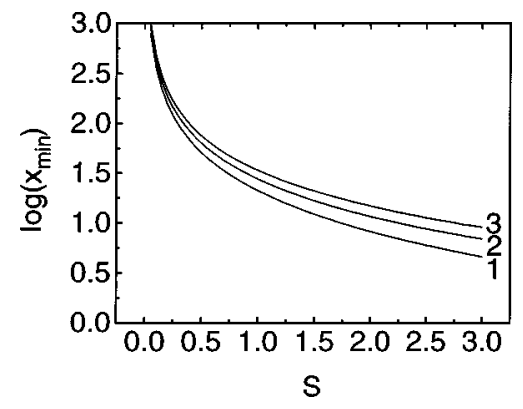

FIG. 4. Plot of $\log _{10}\left(x_{\min }\right)$ vs $S$ for three values of the dislocation core energy. Curves 1,2 , and 3 are for core energies of $E_{c}, 2 E_{c}$, and $3 E_{c}$ where $E_{c}=2 \pi r \gamma_{0}=1.3 \mathrm{eV} / \AA$. 
TABLE I. GaN surface energies in the Ga-rich limit.

\begin{tabular}{lcc}
\hline \hline \multicolumn{1}{c}{ Surface } & Energy $\left(\mathrm{meV} / \AA^{2}\right)$ & Surface structure \\
\hline$(10 \underline{10})$ & 110 & Ga terminated $^{\mathrm{a}}$ \\
$(10 \underline{10})$ & 118 & stoichiometric $^{\mathrm{a}}$ \\
$(001)$ & 125 & $1 \times 4 \mathrm{Ga}$ tetramer \\
$\frac{1}{2}\{(0001)+(000 \underline{1})\}$ & 123 & $2 \times 2 \mathrm{Ga}$ adatom $+1 \times 1 \mathrm{Ga}$ adlayer \\
$\frac{1}{2}\{(10 \underline{1} 1)+(10 \underline{1})\}$ & 125 & $1 \times 1 \mathrm{Ga}$ adlayer $+1 \times 1 \mathrm{Ga}$ adlayer \\
\hline \hline
\end{tabular}

${ }^{\mathrm{a} R e f .} 8$.

${ }^{\mathrm{b}}$ Ref. 9.

an increase of the core energy by a factor of three $(\lambda$ $\rightarrow 3 \lambda$ ) we obtain a rather generous upper limit for the equilibrium pit diameter of $2 a \sim 60 \AA$. A quantitative experimental study of the equilibrium pit size in unalloyed GaN films is not currently available. Indeed, pit formation is rarely seen in such films and is most often associated with the presence of In in the films.

We have shown that In prefers to occupy (1011) surface sites rather than bulk sites. Because the In-N bond is longer and weaker than the $\mathrm{Ga}-\mathrm{N}$ bond, the system can lower its energy by interchanging fourfold coordinated In atoms in the bulk with onefold and twofold coordinated Ga atoms on the (1011) surface. Moving an In from a bulk site to a $B 2$ site lowers the energy by $1.5 \mathrm{eV}$. Moving the In from a bulk site to a $T 1$ site lowers the energy by $2.0 \mathrm{eV}$. Thus, under growth conditions for which In is also incorporated in the bulk, the formation energy of an In-terminated surface will be lower than that of the Ga-terminated surface by about $3.5 \mathrm{eV}$ per $1 \times 1$ unit cell. Since the area of the $1 \times 1$ unit cell is $18.6 \AA^{2}$, the formation energy may be reduced by up to $188 \mathrm{meV} / \AA^{2}$. Thus, when In is present in the epitaxial film, $S$ may be reduced to very low values $(S<1)$, and as seen in Fig. 4 this would lead to the formation of very large pits. We therefore propose that the formation of low energy In-terminated surfaces drives the growth of large pits.

Finally, we propose a mechanism to explain chemical ordering in InGaN alloys. Electron diffraction analysis ${ }^{6,7}$ reveals that in some cases the (0001) cation planes are alternately $\mathrm{Ga}$ rich or rich along the [0001] direction. Studies of zinc-blende alloys have shown that bulk ordering need not be a consequence of bulk thermodynamics. ${ }^{14}$ In fact, our calculations for $\operatorname{In}_{0.5} \mathrm{Ga}_{0.5} \mathrm{~N}$ compounds show that the ordered bulk compound is not stable with respect to mixing of In and $\mathrm{Ga}$ within a given (0001) plane. However, one can explain the ordering within a model wherein growth occurs by attachment of atoms at step edges or small (1011) facets. As shown in Fig. 5 a bilayer step of height $c$ has the same local atomic structure as a (1011) microfacet. We have shown that $\operatorname{In}(\mathrm{Ga})$ atoms preferentially occupy adlayer sites in which they make one(two) bond(s) to the $\mathrm{N}$ atoms. ${ }^{15}$ The energy difference between the favorable and unfavorable

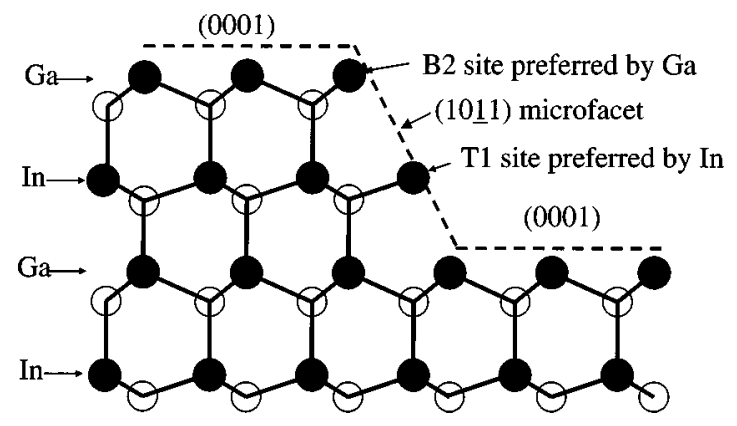

FIG. 5. Representation of a step of height $c$ on $\mathrm{GaN}(0001)$. At such a step the cations attach at either $B 2$ or $T 1$ sites. The Ga binds preferentially in $B 2$ sites while the In binds in $T 1$ sites, and this leads to the formation of alternately $\mathrm{Ga}$ and In-rich (0001) planes as the steps flow across the surface.

configurations is $0.5 \mathrm{eV}$, a value that is large compared to the growth temperature $\left(k T_{\text {growth }}=0.09 \mathrm{eV}\right)$. Consequently as the steps sweep across the surface, Ga incorporation occurs mainly on the $\mathrm{Ga}$ planes while In incorporation occurs mainly on the In planes indicated in Fig. 5. This ordering mechanism will also be operative for single steps of height $c / 2$ provided there is sufficient surface mobility for In to sample the two inequivalent types of steps. One type of step will exhibit $B 2$ sites while the other type exhibits $T 1$ sites. We emphasize that the same mechanism works to explain ordering on the (0001) face of InGaN. In this case our calculations for (1011) surfaces indicate the twofold(threefold) coordinated sites at the steps on (0001) are preferentially occupied by $\operatorname{In}(\mathrm{Ga})$.

${ }^{1}$ A. R. Smith, R. M. Feenstra, D. W. Greve, J. Neugebauer, and J. E. Northrup, Phys. Rev. Lett. 79, 3934 (1997).

${ }^{2}$ S. Kitamura, K. Hiramatsu, and N. Sawaki, Jpn. J. Appl. Phys., Part 2 34, L1184 (1995)

${ }^{3}$ O.-H. Nam, M. D. Bremser, B. L. Ward, R. J. Nemanich, and R. F. Davis, Jpn. J. Appl. Phys., Part 2 36, L532 (1997).

${ }^{4}$ Y. Chen, T. Takeuchi, H. Amano, I. Akasaki, N. Yamada, Y. Kaneko, and S. Y. Wang, Appl. Phys. Lett. 72, 710 (1998).

${ }^{5}$ X. H. Wu, C. R. Blass, A. Abare, M. Mack, S. Keller, P. M. Petroff, S. P. DenBaars, J. S. Speck, and S. J. Rosner, Appl. Phys. Lett. 72, 692 (1998).

${ }^{6}$ P. Ruterana, G. Nouet, W. Van der Stricht, I. Moerman, and L. Considine, Appl. Phys. Lett. 72, 1742 (1998).

${ }^{7}$ D. Doppalapudi, S. N. Basu, K. F. Ludwig, Jr., and T. D. Moustakas, J. Appl. Phys. 84, 1389 (1998).

${ }^{8}$ J. E. Northrup and J. Neugebauer, Phys. Rev. B 53, 10477 (1996).

${ }^{9}$ J. Neugebauer, T. K. Zywietz, M. Scheffler, J. E. Northrup, and C. G. Van de Walle, Phys. Rev. Lett. 80, 3097 (1998).

${ }^{10}$ F. C. Frank, Acta Crystallogr. 4, 497 (1951).

${ }^{11}$ J. E. Northrup, R. Di Felice, and J. Neugebauer, Phys. Rev. B 56, 4325 (1997)

${ }^{12}$ J. Elsner, R. Jones, P. K. Sitch, V. D. Porezag, M. Elstner, T. Frauenheim, M. I. Heggie, S. Oberg, and P. R. Briddon, Phys. Rev. Lett. 79, 3672 (1997).

${ }^{13}$ E. Arbel and J. W. Cahn, Surf. Sci. 51, 305 (1975). $S$ is an invariant combination of the energies of the (0001) and (1011) surfaces.

${ }^{14}$ S. Froyen and A. Zunger, Phys. Rev. Lett. 66, 2132 (1991).

${ }^{15}$ This energetic preference is quite general: Our calculations for the (1012) surface indicate that $\mathrm{In}-\mathrm{Ga}$ exchange between $T 1-B 2$ sites is favorable by $0.7 \mathrm{eV}$. 